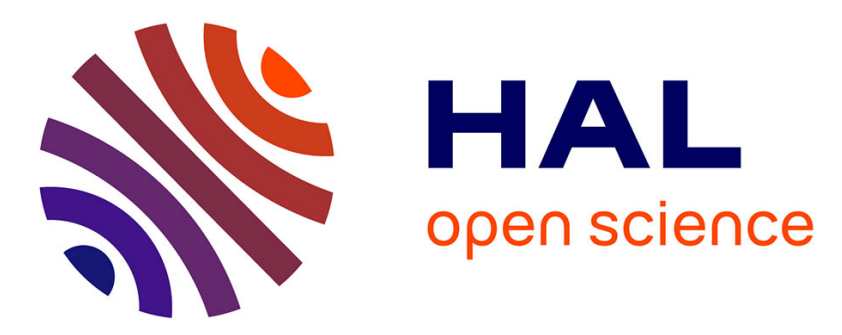

\title{
Anomalous acceleration/heating of charged particles in a random electric field
}

\author{
Vladimir Krasnoselskikh, T. Hada, B. Lefebvre
}

\section{To cite this version:}

Vladimir Krasnoselskikh, T. Hada, B. Lefebvre. Anomalous acceleration/heating of charged particles in a random electric field. The solar wind nine conference, 1999, Nantucket, United States. pp.377-380, 10.1063/1.58776 . insu-02926910

\section{HAL Id: insu-02926910 https://hal-insu.archives-ouvertes.fr/insu-02926910}

Submitted on 1 Sep 2020

HAL is a multi-disciplinary open access archive for the deposit and dissemination of scientific research documents, whether they are published or not. The documents may come from teaching and research institutions in France or abroad, or from public or private research centers.
L'archive ouverte pluridisciplinaire HAL, est destinée au dépôt et à la diffusion de documents scientifiques de niveau recherche, publiés ou non, émanant des établissements d'enseignement et de recherche français ou étrangers, des laboratoires publics ou privés. 


\title{
Anomalous Acceleration/Heating of Charged Particles in a Random Electric Field
}

\author{
V. Krasnoselskikh ${ }^{\mathrm{a}}$, T. Hada ${ }^{\mathrm{b}}$ and B. Lefebvre ${ }^{\mathrm{a}}$ \\ ${ }^{a}$ LPCE/CNRS, 3A av. de la Recherche Scientifique, 45071 Orléans, France and ${ }^{b}$ Earth System Science and Technology, \\ Kyushu University, 6-1 Kasuga-Koen, Kasuga, Fukuoka 816-8580, Japan
}

\begin{abstract}
Acceleration and heating of particles in a given electric field is considered by performing test particle type simulations. The purpose of this study is to identify relationships between statistics of the electric field and those of particles. Electric field is taken to be constant on some randomly chosen intervals and random in other intervals. Potential applications are particle acceleration or heating in quasiparallel shock regions or in solar corona where multiple reconnection sites can occur.
\end{abstract}

\section{INTRODUCTION}

We are interested to study the acceleration and heating of test particles in a given electromagnetic field. Our purpose is to identify the relationship between the statistics of the given field and that of the particle diffusion. We are motivated by the fact that this or quite similar problems appear in several branches of astrophysics and space physics:

a) particle acceleration in the front of collisionless quasi-parallel shock,

b) solar physics (particle acceleration, diffusion) in domains of flares of different scale.

Diffusive shock acceleration mechanism is based on three key elements:

- formation of seed particle population, that is usually assumed to be generated in the shock front

- diffusion of particles that gives rise to the isotropization of their distribution in the reference frame of the upstream and downstream flow keeping their energy constant

- multiple shock front crossings with energy gain after each back and forth trip.

This last element is related to the bulk flow velocity difference between upstream and downstream states of the plasma crossing the shock front. One of the models that was developed to describe this process (1) is based on the presence of structures moving upstream of the shock front with the upstream flow velocity, and downstream with the corresponding Rankine-Hugoniot downstream velocity. This approach qualitatively describes the creation of the seed particle distribution (2), but meets some difficulties in the quantitative description. The presence of non-linear magnetic field structures in the front of quasi-parallel shocks is well documented (see e.g. (3)). Particle diffusion in a gas of such structures assimilated to randomly distributed spheres is a classical mathematical problem of "billiard", and quite well known results of this theory, such as Lyapunov exponents or diffusion coefficient (see e.g. (4)), can be used if the effects of inhomogeneity are neglected. In such a model the interaction with the elementary entities of the turbulence (billiard spheres) is supposed to be elastic. A more detailed analysis of the turbulence in the vicinity of the quasiparallel shock shows that two additional effects can be important for the modeling of these processes:

- the turbulent elements have different scales, that correspond to scaling laws similar to MHD turbulence, though the scaling characteristics are not still established in detail (5)

- these MHD structures can interact with particles in a non-elastic way (6).

The last point is due to the fact that the front regions of the structures are strongly inhomogeneous. In these regions the conditions are favorable for the particle acceleration by means of surfing or gradient drift type mechanisms. Even if the amplitude of the effect will be sufficiently less than in the ramp region of the shock front, the integral effect can be very important because the particle interacts with many such structures, thus during much larger time than with the shock front.

This could be modeled by a random distribution of the spheres having different radii with which particles undergo an elastic collision if the angle between the normal to the sphere surface and velocity vector is less than some critical value, otherwise an electric field assumed to be parallel to the sphere surface acts on the particle. The field in each sphere may for example depend on its 
radius. In this paper, we shall consider the much simpler case (but somehow similar for the physics) of a particle randomly crossing some structures with random electric fields.

Such a description may also apply to particle acceleration and diffusion in multiple domains of solar flares of different scales in the solar corona, that are observed by several experiments (7). Moreover the measurements onboard the GRANAT satellite give clear evidence of the presence of scaling laws for these phenomena $(8,9)$. One of the popular models describing statistical properties of the magnetic fields relies on self-organized criticality $(10,11)$, which results in scaling laws of the magnetic field and associated electric fields (that is, powerlaw dependence of the field intensity on its characteristic scale). The problem of particle diffusion is quite similar to the previously discussed one, because particles can visit many sites, where they undergo the action of electric fields with defined statistical properties. Sites themselves have given statistical properties and distribution.

\section{RANDOM WALKS AND TURBULENCE}

It is well known that Brownian motion gives rise to Gaussian probability distributions with a mean square displacement (msd) linearly dependent on time $\left\langle x^{2}\right\rangle \sim t$ where $\langle. .$.$\rangle denotes ensemble average. However, particle$ diffusion in turbulent flows is known to provide already in the framework of the classical model of Kolmogorov turbulence (12) a quite different diffusive behaviour, which was experimentally discovered by Richardson (13):

$$
\left\langle x^{2}\right\rangle \sim t^{3}
$$

The conventional dependence of the Brownian motion on time is based on the central limit theorem, which states that the limit distribution of the sum of $N$ independant random variables when $N \rightarrow \infty$ is Gaussian, provided the probability distribution of each variable has a finite variance. If it is infinite, then the distribution for the sum converges toward a so-called Lévy-stable law, characterized by a parameter $\beta<2$ which describes the asymptotic decay of the probability density:

$$
p(r) \sim r^{-(1+\beta)}
$$

In some sense Lévy-stable laws have self-similarity properties and exhibit no characteristic scale. The corresponding random walks are called Lévy flights (15)but have the major drawback that the msd of a walker is infinite. However, Schlesinger et al. (16) proposed to impose to the walker a velocity dependent on the step length, in a way compatible with the Kolmogorov scaling for longitudinal velocity fluctuations

$$
\delta V(l) \sim l^{1 / 3}
$$

They showed that this trick makes the msd at a given instant finite, and moreover that the Richardson law of relative diffusion is recovered for $\beta<1 / 3$. These results implicitly assume that the particles are linked to the turbulent flow in all scales and instantly, without any "sliding".

These hypotheses allow to attribute the scaling laws of the turbulent flow to the particle motion under additional condition of the velocity dependence on the scale. There is no consideration of forces acting on the particle in such an approach.

Surprisingly, one can find similar time dependence of the mean square displacement of a walker under the action of a random force in an extremely simple case. Let us consider a discrete random motion under the action of a force verifying

$$
\left\langle F_{i} F_{j}\right\rangle=F^{2} \delta_{i j} .
$$

( $i$ is the $i$-th step). Such a force gives rise to some kind of Brownian motion in the velocity space. Hence the velocity fluctuations are Gaussian and the mean square velocity displacement after $N$ steps:

$$
\left\langle\delta V^{2}\right\rangle \sim N \sim t
$$

This results to the following dependence of the msd under the assumption $V(0)=0$ :

$$
\left\langle x^{2}\right\rangle \sim F^{2} \sum_{n=1}^{N} n^{2}=F^{2} \frac{N(N+1)(2 N+1)}{6} \sim t^{3}
$$

\section{STATEMENT OF PROBLEM}

Above discussions lead to the following questions: how do the statistical properties of fields (this includes the properties of their amplitudes and time/spatial scale distributions) influence the characteristics of the particle diffusion and acceleration processes?

If one takes the equations of motion in their simple form

$$
\frac{d \mathbf{r}}{d t}=\mathbf{v}, \quad \frac{d \mathbf{v}}{d t}=\mathbf{f}
$$

where $f$ has prescribed statistical properties. Clearly, particles statistics will be determined by those of the force. Their time/spatial scales and amplitudes are closely related in models of MHD-type turbulence in the vicinity of the shock front, and in models of multi-scales reconnection sites. 
Short Large Amplitude Magnetic Structures (SLAMS) observed near quasi-parallel shocks have clearly identified scaling laws: larger structures tend to have larger fluctuations of magnetic and associated electric fields. The surfing mechanism, that can be responsible for the non-elastic particle-structure interaction, is based on an electric field component perpendicular to the magnetic field in the front region of the structures, and depends on the magnetic field inhomogeneity.

The amplitude of the electric field in the reconnection site is associated with the energy flux into it, eventually of bursty nature (explosive regime), and may be associated with the amplitude of fluctuating Alfvern velocity in close vicinity of the site. The scale of the acceleration region is assumed to be determined by the Larmor radius of ions in the surrounding background magnetic field (ions are supposed to be magnetized outside but not inside).

Hereafter, statistical properties of the electric fields are determined by the the intervals where they are applied. Several levels of studies, with increasing complexity and sophistication can be considered:

1. The basic equations of motion can be discretized,

$$
\begin{aligned}
& \mathbf{x}(i+1)=\mathbf{x}(i)+\mathbf{v}(i) \\
& \mathbf{v}(i+1)=\mathbf{v}(i)+\mathbf{f}(i)
\end{aligned}
$$

$\mathbf{x}, \mathbf{v}$ being $n$-dimensional position and velocity, and $f$ the normalized force, which may depend on $\mathbf{v}$ as well (Lorentz force).

From the point of view of numerics, these equations are equivalent to continous ones 3 as long as the time step used is taken much shorter than the correlation time scale of the forcing. The situation may be different if $\mathbf{f}$ depends on $\mathbf{x}$, in which case the effect of the particle-"wave" resonance may become quite significant.

One can also include the effect of relativity, as necessity arises.

2. The forcing can be given in a variety of ways:

(a) A random force with $\langle f\rangle=0$ and $\left\langle f^{2}\right\rangle=1$ (since the basic equations of motion are linear, $f$ can always be put in this way), corresponding to Brownian motion.

(b) An "intermittent force" can be constructed in a simple way: time intervals without forcing alternate with intervals of nonzero $\left\langle f^{2}\right\rangle$. Intervals may have different lengths. We shall assume here

$$
\begin{aligned}
f(t) & \neq 0 \text { for } m T<t<(m+p) T \\
f & =0 \text { otherwise }
\end{aligned}
$$

where $m$ is an integer and $p$ is the fraction of time with nonzero $f$. The simplest is the case with $T$ and $p$ both constant, but either $T$ or $p$ can be made variable with given distribution. (c) Further complication is given by allowing the magnitude of $f$ to depend on the time interval $T$ which models more realistic situations in the solar wind, such as SLAMS. Also, one can specify some finite value to $f=$ $f_{0}$ in the period of time $(m+1-p) T<t<(m+1) T$, in order to look at the effect of constant acceleration. However it is required that on a global time scale $\left\langle f_{0}\right\rangle=0$.

(d) $f$ can be taken as a synthetic "multi-affine" field, either in 1,2 , or $3 \mathrm{~d}$ with prescribed multifractal properties. There exist for quite a long time rather successful techniques to reproduce random variables with similar properties to those of measured quantities such as the energy dissipation rate in a turbulent fluid (17).

3. The one dimensional case is already an adequate starting point exhibiting interesting phenomena and allowing several variations (as discussed previously). The $2 \mathrm{~d}$ case allows more effects, as the divergence of trajectories, Fermi acceleration, acceleration due to the gradient$B$ force, etc. It has more direct application to the acceleration in the vicinity of planetary bow shocks.

4. The obtained time series can be analyzed by the now-standard techniques of turbulence and multifractal analysis, such as structure functions and the $f(\alpha)$ spectrum. We should also evaluate the particle diffusion properties, including the energy diffusion (in 1,2,3d cases) and the pitch-angle diffusion (in 2,3d).

\section{PRELIMINARY RESULTS}

In order to launch the above described project we have computed several different simulation runs, with some variety of models and different parameters. The Figures are available on the $C D$. All the runs presented in Figures 1 to 7 represent one-dimensional cases with the equations of motion 4 and 5. Figs. 8 to 13 are two-dimensional.

The total length of time steps, $N T$, is $10^{5}$. The panels in the figures show $F, v$, and $x$ as functions of time. Upper panels of figures B represent the structure functions, $S_{q}(\tau)$, for $F, v, x$, respectively, as function of the correlation time $\tau$ (horizontal axis) for several order $q$. For a given time series $G(t)$, the structure function of order $q$ is defined as

$$
S_{q}(\tau)=\left\langle|G(t+\tau)-G(t)|^{q}\right\rangle
$$

and is supposed in case of a "turbulent" field to behave as a power-law in some range of $\tau$

$$
S_{q}(\tau) \sim \tau^{\zeta(q)}
$$

The exponent is related to the fractal properties of the turbulence (18). Intermittency corresponds to a non-linear dependence of $\zeta$ on $q$ and is associated to multifractality (for a homogeneous turbulent field is linear on $q$ ). In 
practice scaling laws are better detected when the structure functions $S_{q}$ are normalized to some $S_{p}$

$$
S_{q}^{(n o r m)}(\tau)=\frac{S_{q}(\tau)}{\left(S_{2}(\tau)\right)^{q / 2}}
$$

If the particle trajectories has the intermittency property these functions can provide quantitative characterization of it. However the detailed analysis of these characteristics are beyond the scope of our paper and will be published elsewhere.

\section{FIGURE CAPTIONS}

Most of the Figures show structure functions of index $p$ ranging from 1 to 5 . They are noted respectively by the following signs: $1-+, 2-*, 3-x, 4-\Delta, 5$ - square.

Fig. 1. The run with a uniform distribution of $F$ in the interval $[-1,1]$.

Fig. 2. The force is given intermittency by $T=1000$ and $p=0.3$ in equation 7 .

Fig. 3. $T$ has a probability density

$$
\begin{aligned}
& \rho(T)=\left(\frac{T}{T_{0}}\right)^{-\alpha} \quad \text { if } T_{0}<T<T_{1} \\
& \rho(T)=0 \text { otherwise }
\end{aligned}
$$

where in this particular run, $T_{0}=100, T_{1}=10000$, and $g=2$ have been chosen. Otherwise, all the parameters used are the same as previous runs, i.e., $N T=100000$ and $p=0.3$.

Fig. 4. Same as Fig. 3 except that $p=0.7$.

Fig. 5. The amplitude of $F$ is chosen to be proportional to the interval length, $T$. Otherwise, parameters are the same as in Fig. 3.

Fig. 6. Same as Fig. 5 but $p=0.7$.

Fig. 7. Same as Fig. 5 but $p=0.1$.

Runs shown in Fig. 8 to Fig. 13 correspond to the twodimensional case where the force amplitude, $F$, obeys

$$
\begin{aligned}
& \rho(F)=a R F^{-\alpha} \quad \text { if } F_{0}<F<F_{1} \\
& \rho(F)=0 \text { otherwise }
\end{aligned}
$$

where $g$ is the given power law index, $R$ is randomly assigned a value of either 1 or -1 , and $a$ is the normalization constant. This is a rather simple model so its statistical properties may have already been analyzed.

Fig. $8 . g=1$.

Figs. 9 and $10 . g=2$.

Fig. 11. $g=3$ (Lévy flight-like patterns are seen).

Fig. 12. $g=4$.

Fig. 13. The force is given as a Gaussian.

Acknowledgement V.K. and T.H. acknowledge ISSI (Berne) for financial support in the framework of the working group on "Collisionless Shocks".

\section{REFERENCES}

1. Jones, F.C., and D. Ellison, Space Sci. Rev., 58 (1991).

2. Ellison, D., E. Möbius, G. Paschmann, Ap. J., 352, 376-394 (1990).

3. Schwartz, S.J., D. Burgess, W.P. Wilkinson, R.L. Kessel, M. Dunlop, H. Lühr, J. Geophys. Res., 97, A4, 4209-4227 (1992).

4. Chernov N., J. Stat. Phys., 88 1/2, 1-29 (1997).

5. Dudok de Wit, T., and V. Krasnoselskikh, Nonlinear Processes in Geophysics 3, 262-273 (1996).

6. Kucharek, H., and M. Scholer, J. Geophys. Res., 96, N21 (1991).

7. Vilmer, N., G. Trottet, in Proceedings of the CESRA Workshop, "Coronal Physics from Radio and Space Observations", ed. by G. Trottet, Springer, Lecture Notes in Physics, vol. 483 (1997).

8. Crosby, N., M.J. Aschwanden, B.R. Dennis, Solar Physics, 143, 275-299 (1993).

9. Crosby, N., N. Vilmer, N. Lund, R. Sunyaev, Astron. Astrophys., 334, 299 (1998).

10. Lu E.T., and R.J. Hamilton, Ap. J. L., 380, L89 (1991).

11. Vlahos, L., M. Georgoulis, R. Kluiving, and P. Pachos, Astron. and Astrophys., 299, 897 (1995).

12. Kolmogorov, A.N., Dolk. Acad. of Sci. USSR, 30 (1941).

13. Richardson, L.F., Proc. Roy. Soc. London, A110, 33-39 (1926).

14. Lévy P., Théorie de l'Addition des Variables Aléatoires, Gauthier-Villars, Paris (1937).

15. Klafter, J., M.F. Shlesinger and G. Zumofen, Physics Today, 33-39 (1996).

16. Shlesinger, M.F., B.J. West, J. Klafter, Phys. Rev. Lett, 58, N1 I, 1100 (1987).

17. Meneveau, C. and K.R. Sreenivasan, Phys. Rev. Lett., 59, N11, 1424 (1987).

18. Rose, H.A., and P.L. Sulem, J. Physique, 39, 441-484 (1978). 